\title{
Does bipolar disorder have a benign course?
}

Written records have been describing extreme states of emotions since ancient Greece (Angst and Marneros, 2001), but Aretaeus of Cappadocia (which is geographically located in modern Turkey) was probably the first to outline the close relationship between depression and mania nearly 2000 years ago: "I think that melancholia is the beginning and a part of mania... The development of mania is really a worsening of the disease rather than a change into another disease... In most of them the sadness became better after various lengths of time and changed into happiness; the patients then developed a mania" (Angst and Marneros, 2001). Limited progress in our understanding of these extreme states of affect occurred until the mid-1800s, when both German and French physicians suggested that the change from melancholia to mania was not only "usual," but that the continuous cycle between depression, mania, and disease free intervals were the key features of a mental disorder. Jean Pierre Falret named the disorder "folie circulaire" (1851), while Jules Baillarger called it "folie a double-form" (1854). Karl Kahlbaum, working in Germany, supported the French concept of "circular insanity" and his work played a key role in the classification of mental disorders put forward by Emil Kraepelin, which separated manic-depressive insanity from dementia praecox, paranoia, and paraphrenia (Angst and Marneros, 2001). According to Kraepelin, manicdepressive insanity was characterized by periods of melancholia, mania, and normal function. The concept of manic-depressive insanity evolved into that of bipolar disorder and was later incorporated into the Diagnostic and Statistical Manual of Mental Disorders (DSM) and the International Classification of Mental Disorders (ICD). At present, the diagnosis of bipolar disorder requires a current or past episode of mania or mixed state, and the assurance that the changes in mood are not due to other conditions such as schizophrenia (or dementia praecox, as Kraepelin called it) or other psychotic disorders. The underlying assumption of the modern concept of bipolar disorder is that the condition is characterized by nothing else but the episodes of mania and depression.

\section{Function between mood episodes in people with bipolar disorder}

In the late 1990s, several research papers described findings indicating that euthymic adults with bipolar disorder showed evidence of subtle impairments in cognitive function (Ferrier et al., 1999). The concern, in this case, was that these cognitive deficits could potentially compromise the function of patients between the episodes of mood disturbance. Ten years ago, an American study followed adolescents presenting with their first ever hospital admission with mania or a mixed episode (DelBello et al., 2007). The investigators found that about $50 \%$ of participants experienced syndromic recovery within $12-16$ weeks and $86 \%$ within one year. In contrast, only about $17 \%$ of participants experienced functional recovery during the initial 12-16 weeks following admission and $43 \%$ by the end of one year (DelBello et al., 2007). In other words, nearly $60 \%$ of the adolescents with bipolar disorder in this study failed to regain full functional capacity during the year following their first ever admission to hospital. Subsequently, a cross-sectional study that used the Functioning Assessment Short Test (FAST) found that $60 \%$ of the 71 euthymic adults with bipolar disorder were functionally impaired compared with $13 \%$ of the controls (Rosa et al., 2009), suggesting that the typical "disease free interval" of bipolar disorder may not necessarily be "impairment free."

\section{Cognitive function in bipolar disorder, and the risk of dementia and death}

A recent meta-analysis of individual patient data compared the performance of euthymic adults with bipolar disorder with that of controls on 11 cognitive measures derived from tests of verbal learning, trail making, digit span, and/or the Wisconsin Card Sorting Test (Bourne et al., 2013). Bipolar disorder was associated with impairments in all measures, even after the analyses were controlled for the possible confounding effects of age, gender, and premorbid intelligence (Bourne et al., 2013). The authors of this meta-analysis also noticed that some cognitive deficits were associated 
with illness severity, and suggested that they seemed to track the progression of the disorder (Bourne et al., 2013). This raises the question of whether the cognitive and functional deficits observed among euthymic people with bipolar disorder are progressive in nature and may ultimately lead to the development of dementia.

We have recently reported the results of a population-based longitudinal study of men who were aged 65-85 years in 1996. Participants were free of dementia at the beginning of the followup period of 12 years (Almeida et al., 2016b). Of the 37,768 participants, $256(0.7 \%)$ had a recorded diagnosis of bipolar disorder. Compared with other participants, men with bipolar disorder had five times greater prevalence of alcohol-related disorders and $70 \%$ greater odds of other substance use disorder (Almeida et al., 2016b). The hazard ratio of dementia during follow-up was 2.6 times greater in men with than without bipolar disorder (95\% confidence interval: 2.0 to 3.3), and this increase in risk remained even after the analyses were adjusted for age and use of substances, and when death was used as a competing risk (Almeida et al., 2016b). The risk of dementia was particularly high for men with recent (i.e. $<5$ years) history of bipolar disorder, suggesting that changes in the regulation of affect in later life may herald, in at least some cases, the early stages of dementia. A similarly high risk of dementia was observed among men with a longstanding history (i.e. $\geq 15$ years), implying that prolonged exposure to the physiological concomitants of bipolar disorder may facilitate neuronal loss and the development of dementia (Moorhead et al., 2007). Consistent with this idea, a Danish case-register study found that the risk of dementia in bipolar disorder increased with the number of affective episodes (Kessing and Andersen, 2004), and was less marked among patients receiving continuous treatment with lithium (but not with other mood stabilizers) (Kessing et al., 2010).

Bipolar disorder has also been associated with increased mortality (Almeida et al., 2014), including death by suicide (Schaffer et al., 2015). A systematic review and meta-analysis of 31 studies that included over 300,000 people with bipolar disorder found that the condition more than doubled the risk of premature death both from natural and non-natural causes (such as suicide or accidents) (Hayes et al., 2015).

\section{Bipolar disorder: preparing for the future}

One of the features that separated Kraepelin's manic-depressive illness (bipolar disorder) from dementia praecox (schizophrenia) was the recovery of function and absence of residual symptoms between episodes. However, recent data suggest that the euthymic periods are not entirely free of impairments, and that the long-term outcome of people with bipolar disorder is, in at least some cases, associated with cognitive deficits, poor function, and increased risk of dementia and death (Kessing et al., 1999; Bourne et al., 2013; Almeida et al., 2014; Almeida et al., 2016b).

The underlying causes associated with this restricted prognosis of bipolar disorder are not well understood, but could include lifestyle choices, concurrent comorbidities, psychosocial adversity, and sub-optimal access to supportive health services. Regardless of the causes, it is clear that the management of bipolar disorder should not be limited to the acute treatment of the affective episodes, particularly if we wish to improve the long-term outlook and function of these patients as they move toward old age.

As the world's population ages, mental health services for older adults will be responsible for the care on an increasingly larger number of people with bipolar disorder - these services should become actively engaged in the development of effective strategies to improve the clinical outcome of these patients. We will need to progress our understanding of the factors that mediate the age of onset of illness, the overlap between bipolar disorder and dementia in later life, as well as the protective and deleterious risk factors associated with good and poor functional outcomes. In practice, this will probably require the establishment of national and international collaborative networks that follow similar protocols for assessment, modification of risk factors (for example, Almeida et al., 2013; Almeida et al., 2016a), and treatment of adults with bipolar disorder (Sajatovic et al., 2015; Dols et al., 2016). It is time to get moving!

\section{Conflict of interest}

None.

\section{Funding}

None.

\section{Osvaldo P. Almeida}

School of Psychiatry \& Clinical Neurosciences and Western Australian Centre for Health \& Ageing, University of Western Australia 
Department of Psychiatry, Royal Perth Hospital and

Bentley Mental Health Services for Older Adults

Perth, Western Australia, Australia

Email: osvaldo.almeida@uwa.edu.au

\section{References}

Almeida, O. P., Hankey, G. J., Yeap, B. B., Golledge, J., McCaul, K. and Flicker, L. (2013). A risk table to assist health practitioners assess and prevent the onset of depression in later life. Preventive Medicine, 57, 878-882.

Almeida, O. P., Hankey, G. J., Yeap, B. B., Golledge, J., Norman, P. E. and Flicker, L. (2014). Mortality among people with severe mental disorders who reach old age: a longitudinal study of a community-representative sample of 37,892 men. PLoS One, 9, e111882.

Almeida, O. P., McCaul, K., Hankey, G. J., Yeap, B. B., Golledge, J. and Flicker, L. (2016a). Risk of dementia and death in community-dwelling older men with bipolar disorder. British Fournal of Psychiatry, 209, 121-126

Almeida, O. P. et al. (2016b). Reducing depression during the menopausal transition with health coaching: results from the healthy menopausal transition randomised controlled trial. Maturitas, 92, 41-48.

Angst, J. and Marneros, A. (2001). Bipolarity from ancient to modern times: conception, birth and rebirth. Fournal of Affective Disorders, 67, 3-19.

Bourne, C. et al. (2013). Neuropsychological testing of cognitive impairment in euthymic bipolar disorder: an individual patient data meta-analysis. Acta Psychiatrica Scandinavica, 128, 149-162.

DelBello, M. P., Hanseman, D., Adler, C. M., Fleck, D. E. and Strakowski, S. M. (2007). Twelve-month outcome of adolescents with bipolar disorder following first hospitalization for a manic or mixed episode. American fournal of Psychiatry, 164, 582-590.
Dols, A. et al. (2016). Do current national and international guidelines have specific recommendations for older adults with bipolar disorder? A brief report. International fournal of Geriatric Psychiatry. doi:10.1002/gps.4534.

Ferrier, I. N., Stanton, B. R., Kelly, T. P. and Scott, J. (1999). Neuropsychological function in euthymic patients with bipolar disorder. British Fournal of Psychiatry, 175, 246-251.

Hayes, J. F., Miles, J., Walters, K., King, M. and Osborn, D. P. (2015). A systematic review and meta-analysis of premature mortality in bipolar affective disorder. Acta Psychiatrica Scandinavica, 131, 417-425.

Kessing, L. V. and Andersen, P. K. (2004). Does the risk of developing dementia increase with the number of episodes in patients with depressive disorder and in patients with bipolar disorder?. Fournal of Neurology Neurosurgery $\mathbb{E}$ Psychiatry, 75, 1662-1666.

Kessing, L. V., Forman, J. L. and Andersen, P. K. (2010). Does lithium protect against dementia?. Bipolar Disorder, 12, 87-94.

Kessing, L. V., Olsen, E. W., Mortensen, P. B. and Andersen, P. K. (1999). Dementia in affective disorder: a case-register study. Acta Psychiatrica Scandinavica, 100, 176-185.

Moorhead, T. W. et al. (2007). Progressive gray matter loss in patients with bipolar disorder. Biological Psychiatry, 62, 894-900.

Rosa, A. R. et al. (2009). Clinical predictors of functional outcome of bipolar patients in remission. Bipolar Disorder, 11, 401-409.

Sajatovic, M. et al. (2015). A report on older-age bipolar disorder from the international society for bipolar disorders task force. Bipolar Disorder, 17, 689-704.

Schaffer, A. et al. (2015). International society for bipolar disorders task force on suicide: meta-analyses and meta-regression of correlates of suicide attempts and suicide deaths in bipolar disorder. Bipolar Disorder, 17, $1-16$. 